\title{
Usefulness of short-term eltrombopag treatment as a supportive treatment in hepatocellular carcinoma patients with cirrhosis and severe thrombocytopenia: A report of two cases
}

\author{
TAKUMI KAWAGUCHI ${ }^{1,2}$, MASAHITO NAKANO ${ }^{1}$, MANABU SATANI ${ }^{1}$, SHUJI SUMIE $^{1}$, \\ SHINGO YAMADA ${ }^{1}$, KEISUKE AMANO ${ }^{1}$, RYOKO KUROMATSU ${ }^{1}$ and MICHIO SATA ${ }^{1,2}$ \\ ${ }^{1}$ Division of Gastroenterology, Department of Medicine; ${ }^{2}$ Department of Digestive Disease Information and Research, \\ Kurume University School of Medicine, Kurume, Fukuoka 830-0011, Japan
}

Received August 26, 2013; Accepted February 11, 2014

DOI: $10.3892 / \mathrm{ol} .2014 .1976$

\begin{abstract}
Eltrombopag is an oral thrombopoietin (TPO) receptor agonist that increases platelet counts in patients with idiopathic thrombocytopenic purpura and in patients with liver cirrhosis. When cirrhotic patients with thrombocytopenia undergo elective invasive procedures, eltrombopag treatment reduces the requirement for platelet transfusions. However, TPO is known to have proliferative effects on hepatic progenitor cells and hepatic sinusoidal endothelial cells, which indicates that eltrombopag may accelerate tumor progression. Thus, the effect of eltrombopag on hepatocellular carcinoma (HCC) progression is an important issue. The current study describes two cases of HCC with cirrhosis-related thrombocytopenia. A two-week administration of eltrombopag increased platelet counts from 4.8 to $11.3 \times 10^{4} / \mu 1$ in case 1 and 4.5 to $23.2 \times 10^{4} / \mu 1$ in case 2 . However, no changes were identified in the serum levels of tumor markers or HCC size following eltrombopag administration in the two cases. These HCCs were curatively treated by radiofrequency ablation without platelet transfusions or serious bleeding. Thus, short-term eltrombopag administration may not accelerate HCC proliferation and may be beneficial for invasive $\mathrm{HCC}$ treatment in cirrhotic patients with thrombocytopenia.
\end{abstract}

\section{Introduction}

Thrombocytopenia is a common complication of liver cirrhosis and is an intractable issue when performing

Correspondence to: $\mathrm{Dr}$ Takumi Kawaguchi, Division of Gastroenterology, Department of Medicine, Kurume University School of Medicine, 67 Asahi-machi, Kurume, Fukuoka 830-0011, Japan

E-mail: takumi@med.kurume-u.ac.jp

Key words: chronic liver disease, thrombopoietin receptor, hepatoma, growth, megakaryocyte growth and development factor invasive procedures $(1,2)$. When cirrhotic patients with severe thrombocytopenia undergo elective invasive procedures for hepatocellular carcinoma (HCC), $\sim 60 \%$ of the patients require treatment for thrombocytopenia, including platelet transfusions, partial splenic embolization or splenectomy (2). However, platelet transfusions carry the risk of anaphylactic shock, infection and transfusion-related acute lung injuries. Partial splenic embolization may lead to splenic abscesses. In addition, splenectomy may result in splenic vein thrombosis and overwhelming post-splenectomy infections. Thus, a non-invasive therapeutic strategy for thrombocytopenia is required for cirrhotic patients with $\mathrm{HCC}$.

Eltrombopag is an oral thrombopoietin (TPO)-receptor (R) agonist that stimulates megakaryocytes and thus, increases platelet counts in patients with idiopathic thrombocytopenic purpura (3). Since decreased plasma levels of TPO, in addition to hypersplenism, are major pathogenic features of cirrhosis-related thrombocytopenia (1), eltrombopag appears to improve thrombocytopenia in cirrhotic patients. Furthermore, eltrombopag has previously been reported to increase platelet counts and thus, enables subsequent interferon treatments and invasive procedures in cirrhotic patients with severe thrombocytopenia (4-6).

TPO is known as a megakaryocyte growth and development factor that leads to megakaryocyte proliferation. In addition, TPO induces the proliferation of various cell types that express the TPO-R. In the liver, the TPO-R occurs in hepatic progenitor cells (7) and hepatic sinusoidal endothelial cells (8). In addition, TPO induces the proliferation of these two cell types $(7,8)$. Since hepatic sinusoidal endothelial cells and hepatic progenitor cells are crucial in the development and progression of $\mathrm{HCC}$, the effect of eltrombopag on the proliferation of HCC is significant.

The current report presents two cases of HCC with cirrhosis-related thrombocytopenia. In these two cases, eltrombopag treatment increased platelet counts without any concomitant changes in the serum levels of tumor markers or HCC size. The HCCs were subsequently treated by curative radiofrequency ablation (RFA) without platelet transfusions or serious bleeding. Patient provided writted informed consent. 


\section{Case report}

Case 1. In 2008, a 65-year-old male patient was referred to the Kurume University Hospital (Kurume, Japan) for the treatment of hepatitis $\mathrm{C}$ virus (HCV)-related liver cirrhosis. The patient had a history of esophageal varices rupture (that occurred in 1993) and HCC, which was treated by transcatheter hepatic arterial chemolipiodolization with embolization in 2007.

The patient was treated with ursodeoxycholic acid, glycyrrhizin and nutritional therapy, including branched-chain amino acid granules. However, in 2009, a regular abdominal ultrasound revealed a hypoechoic lesion with a maximum diameter of $\sim 15 \mathrm{~mm}$ in Couinaud segment 5 of the liver. The lesion showed decreased contrast uptake in the hepatocyte phase as determined by gadolinium-ethoxybenzyl-diethylenetriamine pentaacetic acid (EOB)-enhanced magnetic resonance imaging (MRI) scan (Fig. 1A). Additionally, an aspiration tumor biopsy revealed that the lesion was a well-differentiated HCC. The $\mathrm{HCC}$ was a single nodule and the patient's Child-Pugh score was 7 points. Although RFA was selected as a therapeutic strategy for HCC, the patient's platelet count $\left(4.8 \times 10^{4} / \mu \mathrm{l}\right)$ revealed severe thrombocytopenia.

The risks and benefits of participation in the phase II study were explained to the patient (5); this study aimed to investigate the efficacy and safety of eltrombopag in patients with thrombocytopenia. Written informed consent was obtained and $25.0 \mathrm{mg}$ eltrombopag was subsequently administered to the patient. The patient's platelet count increased from 4.8 to $11.3 \times 10^{4} / \mu 1$ during the administration of eltrombopag (Fig. 2A; gray area). Following the termination of eltrombopag treatment, the platelet count continued to increase to $21.7 \times 10^{4} / \mu 1$ at three weeks since the initiation of eltrombopag treatment. No significant changes were observed in the serum levels of $\alpha$-fetoprotein and des- $\gamma$-carboxy prothrombin (Table I) or HCC size (Fig. 1B). The HCC was curatively treated by RFA without platelet transfusion or serious bleeding at three weeks following initiation of the eltrombopag treatment.

Case 2. In 2005, a 61-year-old female patient was referred to the Kurume University Hospital for the treatment of HCV-related liver cirrhosis. The patient was treated with ursodeoxycholic acid and glycyrrhizin. However, the hepatic fibrosis gradually progressed. In 2009, a regular abdominal ultrasound revealed a hyperechoic lesion with a maximum diameter of $\sim 7 \mathrm{~mm}$ in Couinaud segment 4 of the liver. An EOB-MRI scan revealed contrast enhancement of the lesion during the early phase (Fig. 1C). The lesion was subsequently diagnosed as a well-differentiated $\mathrm{HCC}$ via an aspiration tumor biopsy. The HCC was a single nodule and the patient's Child-Pugh score was 8 points. RFA was selected as a therapeutic strategy for the HCC, however, the patient's platelet count decreased to $4.5 \times 10^{4} / \mu 1$.

The risks and benefits of participation in the phase II study were explained as previously described (5). Written informed consent was obtained and $25.0 \mathrm{mg}$ eltrombopag was subsequently administered to the patient. The platelet count increased from 4.5 to $23.2 \times 10^{4} / \mu$ l during eltrombopag treatment (Fig. 2B; gray area). Following the termination of eltrombopag treatment, the platelet count increased further to $38.7 \times 10^{4} / \mu \mathrm{l}$ (three weeks since the initiation of eltrombopag treatment).
No significant changes were observed in the serum levels of $\alpha$-fetoprotein and des- $\gamma$-carboxy prothrombin (Table I) or in the HCC size (Fig. 1B). At three weeks following the initiation of eltrombopag treatment, the HCC was curatively treated by RFA without platelet transfusion or serious bleeding.

\section{Discussion}

The effect of eltrombopag on the progression of malignant neoplasms is an important issue. The current report presents two cases of HCC with cirrhosis-related severe thrombocytopenia. The administration of eltrombopag increased the platelet count in these two cases without inducing significant changes in the serum levels of tumor markers or HCC size. These HCCs were successfully treated by RFA without platelet transfusions or serious bleeding. The observations from these cases may indicate that the short-term administration of eltrombopag does not affect the progression of HCC and may be useful when administered prior to invasive procedures in cirrhotic patients with thrombocytopenia.

In the present study, eltrombopag treatment significantly increased platelet count, which subsequently resulted in successful RFA therapy and the avoidance of platelet transfusions. These beneficial observations are consistent with those of previous reports. Eltrombopag has previously been shown to improve thrombocytopenia and permit interferon therapy in patients with cirrhosis that is associated with HCV infection (6). More recently, eltrombopag has been reported to reduce the requirement for platelet transfusions in cirrhotic patients who have undergone elective invasive procedures (4). The study included patients with HCC, however, no monitoring of tumor markers or HCC size was performed, which was conducted in the present study. Thus, eltrombopag may replace platelet transfusions, partial splenic embolization or splenectomy when elective invasive procedures are performed on cirrhotic patients with thrombocytopenia.

In the current two cases, thrombosis did not develop during or following eltrombopag treatment. However, certain previous clinical trials have reported that invasive procedures increase the risk for the development of thrombosis, even in cirrhotic patients with thrombocytopenia $(4,5)$. The risk is proportional to the increasing platelet counts of $>200,000 / \mathrm{mm}^{3}$, which was avoided in the present study by close monitoring of the patients. Although the reason for the development of thrombosis remains unclear, von Willebrand factor levels have been found to increase as an adaptive mechanism in response to reduced platelet adhesion abilities in cirrhotic patients (9). Additionally, an increase was identified in the platelet formation of isoprostanes, which promote platelet activation via the upregulation of proaggregatory factors in cirrhotic patients $(10,11)$. Alternatively, impaired liver function may be involved in the development of thrombosis, since the majority of eltrombopag is eliminated in the liver (12). Higher plasma concentrations of eltrombopag were observed in patients with Child-Pugh class B when compared with patients with Child-Pugh class A (5). Thus, patients must be vigilant for signs of thrombosis, in particular those with advanced liver cirrhosis, during eltrombopag treatment.

Hepatic progenitor cells and hepatic sinusoidal endothelial cells express TPO-R, in addition TPO induces the proliferation 


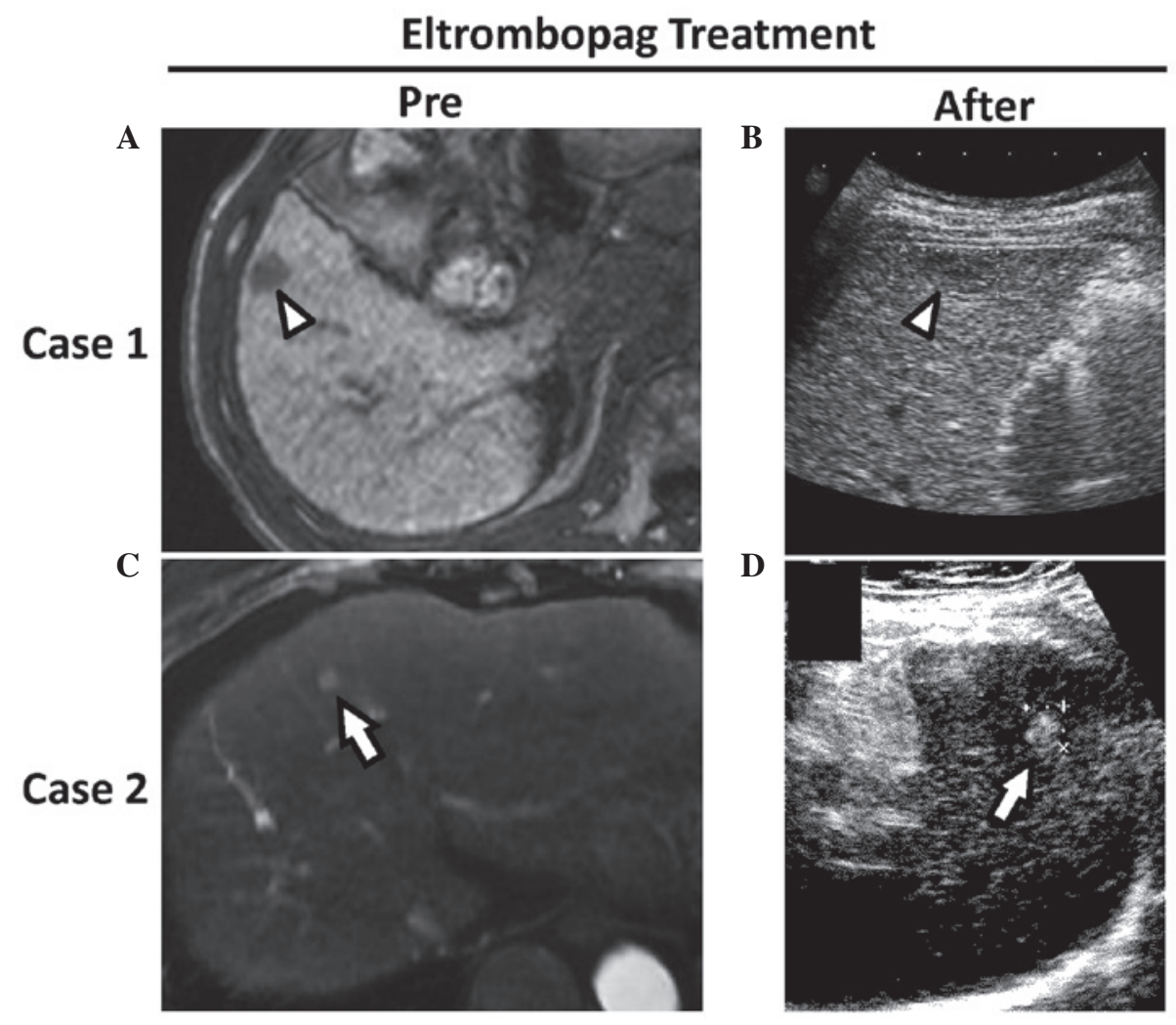

Figure 1. Effect of eltrombopag on HCC size. (A) MRI scan showed a space-occupying lesion with a maximum diameter of $\sim 15 \mathrm{~mm}$ in Couinaud segment 5 of the liver. The lesion showed decreased contrast uptake in the hepatocyte phase following injection of EOB. (B) Two weeks following the eltrombopag treatment, ultrasonography showed a hypoechoic lesion with a maximum diameter of $\sim 15 \mathrm{~mm}$ in Couinaud segment 5 of the liver. (C) MRI scan showed a space-occupying lesion with a maximum diameter of $\sim 7 \mathrm{~mm}$ in Couinaud segment 4 of the liver. The lesion showed contrast enhancement in the early phase following injection of EOB. (D) Two weeks following the eltrombopag treatment, ultrasonography showed a hyperechoic lesion with a maximum diameter of $\sim 8 \mathrm{~mm}$ in Couinaud segment 4 of the liver. HCC, hepatocellular carcinoma; MRI, magnetic resonance imaging; EOB, gadolinium-ethoxybenzyl-diethylenetriamine pentaacetic acid.

A

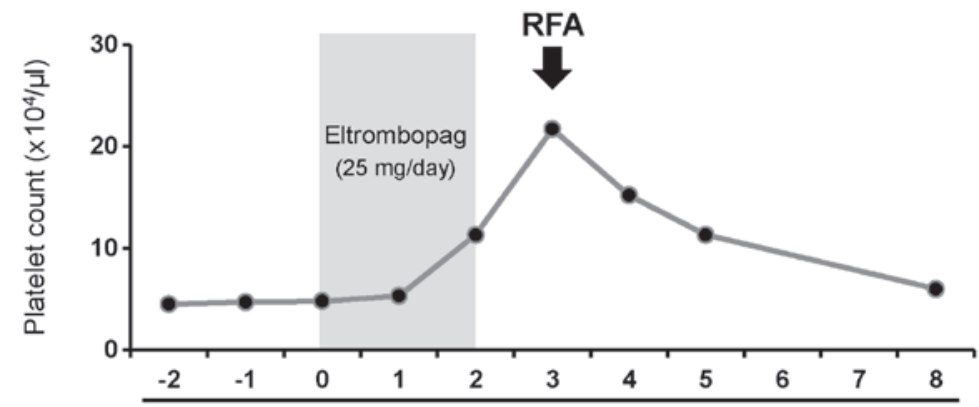

Week(s) after the initiation of eltrombopag treatment

B

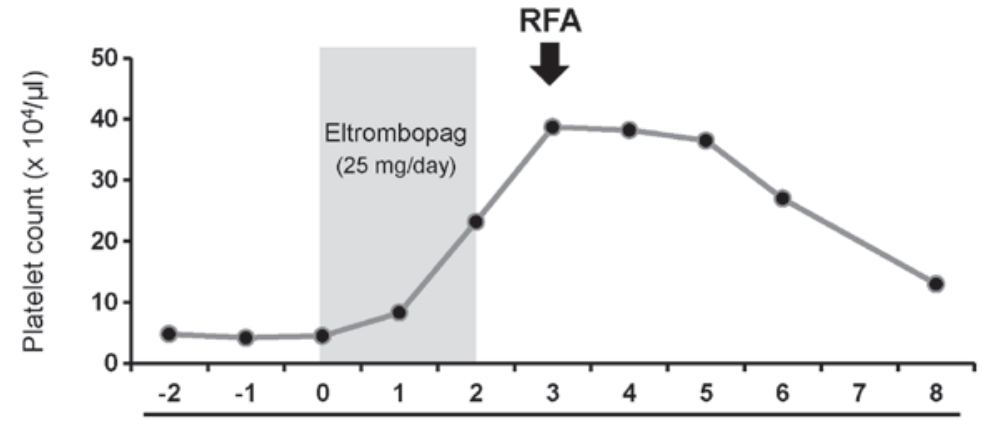

Week(s) after the initiation of eltrombopag treatment

Figure 2. Changes in platelet count following the initiation of eltrombopag treatment. (A) In case 1 , the platelet count increased from 4.8 to $11.3 \times 10^{4} / \mu 1$ during eltrombopag treatment (gray area). Following the termination of eltrombopag treatment, the platelet count increased to $21.7 \times 10^{4} / \mu 1$ (three weeks since the initiation of eltrombopag treatment). (B) In case 2, the platelet count increased from 4.5 to $23.2 \times 10^{4} / \mu 1$ during eltrombopag treatment (gray area). Following the termination of eltrombopag treatment, the platelet count increased further to $38.7 \times 10^{4} / \mu 1$ (three weeks since the initiation of eltrombopag treatment). 
Table I. Changes in biochemical parameters during the course of eltrombopag treatment.

\begin{tabular}{|c|c|c|c|c|c|c|c|}
\hline \multirow[b]{3}{*}{ Biochemical parameter } & \multirow[b]{3}{*}{$\begin{array}{l}\text { Reference } \\
\text { value }\end{array}$} & \multicolumn{6}{|c|}{ Following eltrombopag initiation } \\
\hline & & \multicolumn{3}{|c|}{ Case 1} & \multicolumn{3}{|c|}{ Case 2} \\
\hline & & Pre & Week 2 & Week 3 & Pre & Week 2 & Week 3 \\
\hline Red blood cells, $\times 10^{4} / \mathrm{mm}^{3}$ & $430-570$ & 383 & 368 & 352 & 394 & 369 & 358 \\
\hline Hemoglobin, g/dl & $14.0-18.0$ & 12.5 & 11.7 & 11.5 & 13.1 & 12.3 & 12.1 \\
\hline White blood cells, $/ \mathrm{mm}^{3}$ & $4000-9000$ & 2200 & 2500 & 2600 & 3800 & 4200 & 5200 \\
\hline Platelets, $\times 10^{4} / \mathrm{mm}^{3}$ & 13-36 & 4.8 & 11.3 & 21.7 & 4.5 & 23.2 & 38.7 \\
\hline Aspartate transaminase, $\mathrm{U} / \mathrm{l}$ & $13-33$ & 102 & 67 & 60 & 46 & 35 & 36 \\
\hline Alanine aminotransferase, $\mathrm{U} / \mathrm{l}$ & $8-42$ & 96 & 64 & 49 & 35 & 24 & 22 \\
\hline Lactate dehydrogenase, U/1 & $119-229$ & 285 & 216 & 249 & 298 & 344 & 341 \\
\hline Alkaline phosphatase, U/l & $115-359$ & 352 & 372 & 339 & 278 & 245 & 301 \\
\hline$\gamma$-glutamyl transpeptidase, $\mathrm{U} / \mathrm{l}$ & $10-47$ & 29 & 28 & 27 & 25 & 27 & 39 \\
\hline Total protein, g/dl & $6.70-8.30$ & 8.09 & 7.74 & 7.59 & 7.71 & 7.17 & 7.34 \\
\hline Albumin, g/dl & $4.00-5.00$ & 2.83 & 2.69 & 2.59 & 3.23 & 2.91 & 2.87 \\
\hline Total bilirubin, mg/dl & $0.30-1.50$ & 1.19 & 0.99 & 0.64 & 2.09 & 1.99 & 1.40 \\
\hline Prothrombin activity, \% & $60-130$ & 64 & 59 & 69 & 70 & 66 & 73 \\
\hline Prothrombin activity, INR & $0.87-1.25$ & 1.32 & 1.40 & 1.27 & 1.25 & 1.29 & 1.22 \\
\hline $\mathrm{BUN}, \mathrm{mg} / \mathrm{dl}$ & $8.0-22.0$ & 22.6 & 21.5 & 25.4 & 15.0 & 15.3 & 20.2 \\
\hline Creatinine, $\mathrm{mg} / \mathrm{dl}$ & $0.60-1.10$ & 1.00 & 0.96 & 1.21 & 0.64 & 0.68 & 0.81 \\
\hline Sodium, mEq/l & 138-146 & 137 & 136 & 136 & 139 & 138 & 134 \\
\hline Child-Pugh score & & 7 & 7 & 7 & 8 & 8 & 7 \\
\hline MELD score & & 10 & 10 & 11 & 12 & 12 & 10 \\
\hline$\alpha$-fetoprotein, $\mathrm{ng} / \mathrm{ml}$ & $<8.7$ & 23.0 & 21.8 & 26.2 & 27.8 & 30.5 & 23.9 \\
\hline Des- $\gamma$-carboxy prothrombin, $\mathrm{mAU} / \mathrm{ml}$ & $<40$ & 16 & 14 & 20 & 45 & 35 & 30 \\
\hline
\end{tabular}

BUN, blood urea nitrogen; MELD, model for end-stage liver disease; INR, international normalized ratio.

of these two types of cells $(7,8)$. Furthermore, TPO-R is expressed in hepatoma cell lines, such as Huh7, Hep3B and HepG2 $(13,14)$; these observations indicate that eltrombopag may accelerate the progression of HCC. Although eltrombopag has been administered to treat tumor-bearing patients $(4,15)$, the effect of eltrombopag on tumor progression has not previously been reported. In the present study, it was demonstrated that short-term eltrombopag treatment did not accelerate the progression of HCC. Although, from the results of the two cases the possibility that long-term administration of eltrombopag may accelerate tumor progression cannot be dismissed; however, subsequent findings may support the hypothesis that short-term administration of eltrombopag does not accelerate the proliferation of $\mathrm{HCC}$.

TPO-R expression has previously been found to be lower in hepatoma cell lines compared with expression in primary hepatocytes (14) and HCC tissues do not express TPO-R (13). In addition, TPO does not activate the extracellular signalregulated kinases $1 / 2$ or the signal transducers and activators of transcription 3 and 5 pathways, or affect the proliferation, migration or invasion of Huh7 cells (14). Furthermore, TPO does not upregulate Bax, Bcl-2 or cleaved caspase 3 and does not induce anti-apoptotic effects in Huh7 cells (14). Finally, in a previous xenograft experiment, no significant differences were identified in the tumor volume, tumor appearance and histological morphology of Huh7 cells that were treated with or without TPO (14). Thus, the short-term administration of eltrombopag may not accelerate tumor progression.

In conclusion, the present study described two cases of HCC with cirrhosis-related thrombocytopenia. Eltrombopag treatment increased the platelet count in the two cases and resulted in successful RFA treatments without platelet transfusions or serious bleeding. Additionally, no changes were observed in the serum levels of tumor markers or HCC size. Although eltrombopag is not currently used for the treatment of thrombocytopenia in patients with chronic liver disease undergoing invasive procedures, the observations of the present study indicated that the short-term administration of eltrombopag may not accelerate the progression of $\mathrm{HCC}$ and may be beneficial for HCC treatment in cirrhotic patients with thrombocytopenia.

\section{Acknowledgements}

The authors would like to thank Professor Nezam H. Afdhal (Harvard Medical School, Boston, USA) for his helpful comments.

\section{References}

1. Afdhal N, McHutchison J, Brown R, et al: Thrombocytopenia associated with chronic liver disease. J Hepatol 48: 1000-1007, 2008. 
2. Kawaguchi T, Kuromatsu R, Ide T, et al: Thrombocytopenia, an important interfering factor of antiviral therapy and hepatocellular carcinoma treatment for chronic liver diseases. Kurume Med J 56: 9-15, 2009.

3. Bussel JB, Cheng G, Saleh MN, et al: Eltrombopag for the treatment of chronic idiopathic thrombocytopenic purpura. N Engl J Med 357: 2237-2247, 2007.

4. Afdhal NH, Giannini EG, Tayyab G, et al; ELEVATE Study Group: Eltrombopag before procedures in patients with cirrhosis and thrombocytopenia. N Engl J Med 367: 716-724, 2012.

5. Kawaguchi T, Komori A, Seike M, et al: Efficacy and safety of eltrombopag in Japanese patients with chronic liver disease and thrombocytopenia: a randomized, open-label, phase II study. J Gastroenterol 47: 1342-1351, 2012.

6. McHutchison JG, Dusheiko G, Shiffman ML, et al; TPL102357 Study Group: Eltrombopag for thrombocytopenia in patients with cirrhosis associated with hepatitis C. N Engl J Med 357: 2227-2236, 2007.

7. Schmelzer E, Deiwick A, Bruns H, Fiegel HC and Bader A: Thrombopoietin is a growth factor for rat hepatic progenitors. Eur J Gastroenterol Hepatol 20: 209-216, 2008.

8. Cardier JE and Dempsey J: Thrombopoietin and its receptor c-mpl, are constitutively expressed by mouse liver endothelial cells: evidence of thrombopoietin as a growth factor for liver endothelial cells. Blood 91: 923-929, 1998.
9. Lisman T, Bongers TN, Adelmeijer J, et al: Elevated levels of von Willebrand Factor in cirrhosis support platelet adhesion despite reduced functional capacity. Hepatology 44: 53-61, 2006.

10. Basili S, Raparelli V, Riggio O, et al; CALC Group: NADPH oxidase-mediated platelet isoprostane over-production in cirrhotic patients: implication for platelet activation. Liver Int 31: 1533-1540, 2011.

11. Violi F and Pignatelli P: Eltrombopag before procedures in patients with cirrhosis and thrombocytopenia. N Engl J Med 367: 2056, 2012.

12. Haselboeck J, Kaider A, Pabinger I and Panzer S: Function of eltrombopag-induced platelets compared to platelets from control patients with immune thrombocytopenia. Thromb Haemost 109: 676-683, 2013

13. Columbyova L, Loda M and Scadden DT: Thrombopoietin receptor expression in human cancer cell lines and primary tissues. Cancer Res 55: 3509-3512, 1995.

14. Nozaki R, Murata S, Nowatari T, et al: Effects of thrombopoietin on growth of hepatocellular carcinoma: Is thrombopoietin therapy for liver disease safe or not? Hepatol Res 43: 610-620, 2012.

15. Chawla SP, Staddon A, Hendifar A, Messam CA, Patwardhan R and Kamel YM: Results of a phase I dose escalation study of eltrombopag in patients with advanced soft tissue sarcoma receiving doxorubicin and ifosfamide. BMC Cancer 13: 121, 2013. 